TRABAJOS ORIGINALES

\title{
Diversidad y abundancia de primates en bosques de baja y alta perturbación humana de Los Chilchos, Amazonas, Perú
}

\author{
Diversity and abundance of primates in low and high human disturbance forests of Los \\ Chilchos, Amazonas, Peru
}

\author{
1 Universidad Nacional Mayor de San Marcos, Facultad de Ciencias Biológicas, Lima, Perú. \\ 2 Universidad Nacional de la Amazonía Peruana, Iquitos, Perú. \\ 3 Asociación para la Conservación de Primates Amenazados, Iquitos, Perú. \\ 4 Apenheul Primate Conservation Trust (APCT), The Netherlands. \\ 5 Servicio Nacional de Áreas Protegidas por el Estado, Iquitos, Perú. \\ *Autor para correspondencia \\ Email Rolando Aquino: raquinoy@unmsm.edu.pe; raquinoy2005@yahoo.es \\ Email Luis López: luislopezramirez24@gmail.com \\ Email Johannes Dignum: hdignum@hotmail.com \\ Email Silvia Díaz:silvia.diaz2@unmsm.edu.pe \\ Email Rodrigo Falcón: rodrigofalconayapi@gmail.com
}

Rolando Aquino ${ }^{1 *}$, Luis López ${ }^{2,3}$, Johannes Dignum ${ }^{4}$, Silvia Díaz ${ }^{1}$ y Rodrigo Falcón ${ }^{5}$

\begin{abstract}
Resumen
En los bosques montanos de Los Chilchos habitan cinco especies de primates, cuya situación actual, tamaño de grupo y abundancia son desconocidos, información que es indispensable para el manejo y la formulación e implementación de medidas de conservación. Afín de obtener información sobre los aspectos mencionados, fueron conducidos censos por transecto lineal entre junio y julio del 2017 en bosques de baja y alta perturbación antrópica. Como resultado de los censos fueron registrados 63 grupos pertenecientes a 5 especies. De ellos, 50 grupos correspondieron a los bosques de baja perturbación, siendo Aotus miconax y Ateles belzebuth los más observados con 21 y 17 grupos, respectivamente. Grupos más grandes se observaron en Cebus yuracus (promedio 17.0 \pm 5.0 individuos) y $A$. belzebuth (promedio $14.5 \pm 7.2$ individuos) y más pequeños en $A$. miconax (promedio 2.6 \pm 0.7 individuos). Con excepción de Lagothrix flavicauda, la abundancia relativa y densidad poblacional fueron más altas en bosques de baja perturbación, demostrando así el buen estado de conservación, lo que no ocurre en bosques de alta perturbación. Entre los primates, la densidad más alta fue para $A$. miconax con 26.23 indiv. $/ \mathrm{km}^{2}$ y más baja para Alouatta seniculus con 3.0 indiv. $/ \mathrm{km}^{2}$. Lagothrix flavicauda fue registrada únicamente en bosque de alta perturbación, cuya abundancia relativa fue estimada en $0.1 \mathrm{grupos} / 10 \mathrm{~km}$.
\end{abstract}

Palabras clave: Bosques montanos; nivel de perturbación; especies observadas; tamaño de grupo; densidad poblacional.

Citación:

Aquino R., L. López, J. Dignum, S. Díaz \& R. Falcón. 2018. Diversidad y abundancia de primates en bosques de baja y alta perturbación humana de Los Chilchos, Amazonas, Perú. Revista peruana de biología 25(4): 407 - 416 (Noviembre 2018). doi: http://dx.doi.org/10.15381/rpb.v25i4.15532

Presentado: $\quad 01 / 06 / 2018$

Aceptado: $\quad 02 / 11 / 2018$

Publicado online: 07/12/2018
Fuentes de financiamiento: El presente trabajo se realizó gracias al financiamiento de Primate Action Fund/ Conservation International (6002301); Primate Conservation Inc. (1383); Apenheul Primate Conservation Trust (APCT) The Netherlands.

Información sobre los autores:

RA realizó las coordinaciones con autoridades locales y representantes de la ONG Ucumari, registros de datos en campo, análisis y redacción del manuscrito. LL realizó los registros de campo, apoyó en la redacción del manuscrito. JD realizó los registros de campo, se encargó del apoyo logístico, coordinaciones con ONG Ucumari. SD realizó búsqueda de información bibliográfica, elaboró la base de datos. RF hizo los mapas, apoyó en la interpretación de imagen satelital.

Los autores no incurren en conflictos de intereses. 


\begin{abstract}
In the montane forests of Los Chilchos five species of primates inhabit, whose current situation, group size and abundance are unknown, which are indispensable for the management and formulation and implementation of conservation measures. In order to obtain information on the mentioned aspects, censuses were conducted by linear transect between June and July 2017 in forests of low and high anthropic disturbance. As a result of the censuses, 63 groups belonging to 5 species were registered. Of these, 50 groups corresponded to low disturbance forests, being Aotus miconax and Ateles belzebuth the most observed with 21 and 17 groups, respectively. Larger groups were observed in Cebus yuracus (average $17.0 \pm 5.0$ individuals) and $A$. belzebuth (average $14.5 \pm 7.2$ individuals) and smaller in $A$. miconax (average $2.6 \pm 0.7$ individuals). With exception of Lagothrix flavicauda, the relative abundance and population density were higher in low-disturbance forests, demonstrating thus the good conservation status, which does not occur in high-disturbance forests. Among primates, the highest density was for $A$. miconax with 26.23 indiv. $/ \mathrm{km}^{2}$ and the lowest for Alouatta seniculus with 3.0 indiv. $/ \mathrm{km}^{2}$. Lagothrix flavicauda was recorded only in high disturbance forest, whose relative abundance was estimated at 0.1 groups $/ 10 \mathrm{~km}$.
\end{abstract}

Keywords: Montane forests; disturbance level; species observed; group size; population density.

\section{Introducción}

En los bosques montanos de la Amazonía nororiental habitan no menos de siete especies de primates (Shanee et al. 2013a; Aquino et al. 2017); de ellas, Lagothrix flavicauda y Aotus miconax son endémicas y están categorizadas en el Libro Rojo de la IUCN (2017) como en Peligro Crítico (CR) y Vulnerable $(\mathrm{VU})$, respectivamente; en tanto que las demás especies, entre ellas Ateles belzebuth y Alouatta seniculus, categorizadas como En Peligro (EN) y Preocupación Menor (NT), también tienen como hábitats los bosques inundables y de tierra firme (Aquino \& Encarnación 1994, Defler 2010).

A diferencia de la selva baja, las poblaciones de primates que habitan en bosques montanos están expuestos a la constante reducción y pérdida de sus hábitats por la acelerada deforestación para los fines agrícola, ganadera, floricultura, cultivo ilícito de la coca (Erythroxylum coca) y extracción de recursos forestales (maderas, hojas, lianas, resinas, entre otros), lo que estaría ocasionando una drástica disminución de sus poblaciones. En efecto, la mayoría de los bosques presentes hasta una altitud aproximada de $3400 \mathrm{~m}$ muestran un alto grado de perturbación debido a la deforestación para los fines antes mencionados, constituyéndose así como la principal amenaza para los primates y otros componentes de la fauna silvestre (Leo Luna 1984, Shanee 2012, Shanee et al. 2013a, Aquino et al. 2015). Estas perturbaciones afectan más a los primates que dependen de la vegetación arbórea para sus actividades de locomoción, alimentación, descanso, juegos, entre otros.

En bosques montanos, aun conociendo la existencia de otras especies de primates, los estudios fueron orientadas a L. flavicauda (Leo Luna 1980, 1982, 1984, 1987, Butchart et al. 1995, Cornejo 2007, Shanee et al. 2007a, 2008, Shanee 2011, 2014, Shanee \& Shanee 2011a) y A. miconax (Butchart et al. 1995, Cornejo et al. 2008, Shanee \& Shanee 2011b, 2013a, Shanee et al. 2013b), la mayoría de ellos conducidos en las regiones de Amazonas y San Martín, y están referidos principalmente a aspectos ecológicos, tamaño de grupo, distribución geográfica, entre otros; para el resto de especies, no existe información sobre los aspectos mencionados, salvo los registros de su presencia, en particular de A. belzebuth en la Cordillera de Colán (Mittermeier et al. 1975), Santa Rosa cerca al Abra Patricia (Shanee et al. 2007b), Yambrasbamba, Copallin y Paujil (Shanee et al. (2013a) y recientemente los reportes sobre diversidad y abundancia de primates en Huánuco (Aquino et al. 2015, 2017) y San Martín (Aquino et al. 2017).

En cuanto a Los Chilchos, ubicado en el lado amazónico de la Cordillera nororiental, la única referencia es el inventario de mamíferos realizados por Barrio et al. (2003), quienes reportan seis especies de primates, entre ellas $L$. flavicauda, A. belzebuth y $A$. miconax, pero no proporcionan información acerca del tamańo de grupo y abundancia. Es oportuno precisar, que la tasa de encuentros y en particular la densidad poblacional, son los parámetros fundamentales, no solamente para el manejo de la fauna silvestre y su hábitat, sino también para la formulación e implementación de medidas de conservación (Defler 2003), porque permite conocer el estado actual de sus poblaciones y basados en los resultados tomar decisiones.

La carencia de información sobre el estado actual de los primates que habitan en Los Chilchos fue lo que nos motivó a la conducción de este estudio, cuyo objetivo estuvo orientado a estimar la densidad poblacional en bosques de baja y alta perturbación antrópica. El estudio fue conducido de junio a julio del 2017 en seis sitios de muestreo distribuidos en tres sectores. Los resultados obtenidos se presentan en este reporte.

\section{Área de estudio}

Comprendió los bosques de Los Chilchos perteneciente a la Región Amazonas, donde se encuentra el Área de Conservación Privada (ACP) Los Chilchos, creada en noviembre del 2012 (Resolución Ministerial N³20-2012-MINAM). De acuerdo a informaciones recabadas en la comunidad de Los Chilchos, asentado en la margen derecha del río del mismo nombre, parte de estos bosques ya estaban bajo el cuidado de la comunidad desde muchos años antes a la creación del Área de Conservación Privada en mención, cuyo propósito según manifestaron los entrevistados, era proteger a los componentes de la flora y fauna para el conocimiento de las futuras generaciones de su comunidad, por lo que fue prohibido la extracción de árboles maderables y la caza. 
Afín de comparar la diversidad y abundancia de primates en bosques de alta y baja perturbación humana, en el área de estudio fueron definidos como sectores de muestreo de baja perturbación a Las Hamacas y La Meseta, ubicados entre los ríos Blanco-Lejía y margen derecha del río Lejía, respectivamente, y como de alta perturbación a Las Palmas, localizado entre los ríos Chilchos y Pomalca. En cada uno de estos sectores se establecieron dos sitios de muestreo (Fig. 1), cuyas coordenadas geográficas y altitud se detallan en la Tabla 1. Estos sitios fueron los siguientes:

En el sector de La Meseta, los bosques de ambos lados del río lejía, donde establecimos como sitios de muestreo a Cueva Tranquilo y Maquisapa, el primero localizado al interior del ACP Los Chilchos y el otro fuera de ella. Los bosques aquí presentes podemos considerarlos como de baja perturbación, aun cuando en estos últimos ańos se iniciaron pequeńas deforestaciones, incluso al interior del área de conservación para los fines agropecuarios; no obstante, la fauna mayor está presente, entre ellos los primates como A. belzebuth.
En el sector de Las Hamacas, los bosques existentes entre los ríos Blanco y Lejía pertenecientes al ACP Los Chilchos. Aquí fueron definidos como sitios de muestreo a la Quebrada Shibé y Cueva Lejía. En ambos sitios no se advirtieron mayores perturbaciones que la existencia de senderos para el recorrido de comuneros de Añazco Pueblo y Atuen, por lo que en términos generales podemos considerarlos como de baja perturbación (Fig. 2), en tanto la fauna mayor está presente, entre ellos los primates como A. belzebuth y Alouatta seniculus.

En el sector de Las Palmas, los bosques cercanos al Fundo Pomalca y el poblado de Los Chilchos, ambos ubicados entre los ríos Pomalca y Chilchos. En este sector todavía existen fragmentos o parches de bosque residual relativamente extensos (Fig. 3), pero con un perfil de alta perturbación antrópica, debido a la extracción de árboles maderables con fines comerciales, entre ellos cedro (Cedrella odorata) e ishpingo (Amburana cearensis), así como para la construcción de viviendas y corrales. A ellos se suma la existencia de senderos para el desplazamiento de la gente

Tabla 1. Sitios de muestreo por sectores para los censos de primates en Los Chilchos.

\begin{tabular}{lllcr}
\hline \multirow{2}{*}{ Nivel de perturbación } & \multirow{2}{*}{ Sectores } & Sitios de muestreo & Coordenadas geográficas & Altitud (m) \\
\hline \multirow{2}{*}{ Bajo } & \multirow{2}{*}{ La Meseta } & Cueva Tranquilo & $6^{\circ} 47^{\prime} 03^{\prime \prime} / 77^{\circ} 29^{\prime} 53^{\prime \prime}$ & 2028 \\
& & Maquisapa & $6^{\circ} 48^{\prime} 13^{\prime \prime} / 77^{\circ} 29^{\prime} 29^{\prime \prime}$ & 1857 \\
& \multirow{3}{*}{ Las Hamacas } & Quebrada Shibé & $6^{\circ} 46^{\prime} 52^{\prime \prime} / 77^{\circ} 32^{\prime} 04^{\prime \prime}$ & 1770 \\
& & Cueva Lejia & $6^{\circ} 466^{\prime} 38^{\prime \prime} / 77^{\circ} 30^{\prime} 59^{\prime \prime}$ & 1416 \\
\multirow{2}{*}{ Alto } & \multirow{2}{*}{ Las Palmas } & Pomalca & $6^{\circ} 41^{\prime} 28^{\prime \prime} / 77^{\circ} 38^{\prime} 27^{\prime \prime}$ & 2062 \\
& & Chilchos & $6^{\circ} 43^{\prime} 24^{\prime \prime} / 77^{\circ} 37^{\prime} 43^{\prime \prime}$ & 2096 \\
\hline
\end{tabular}

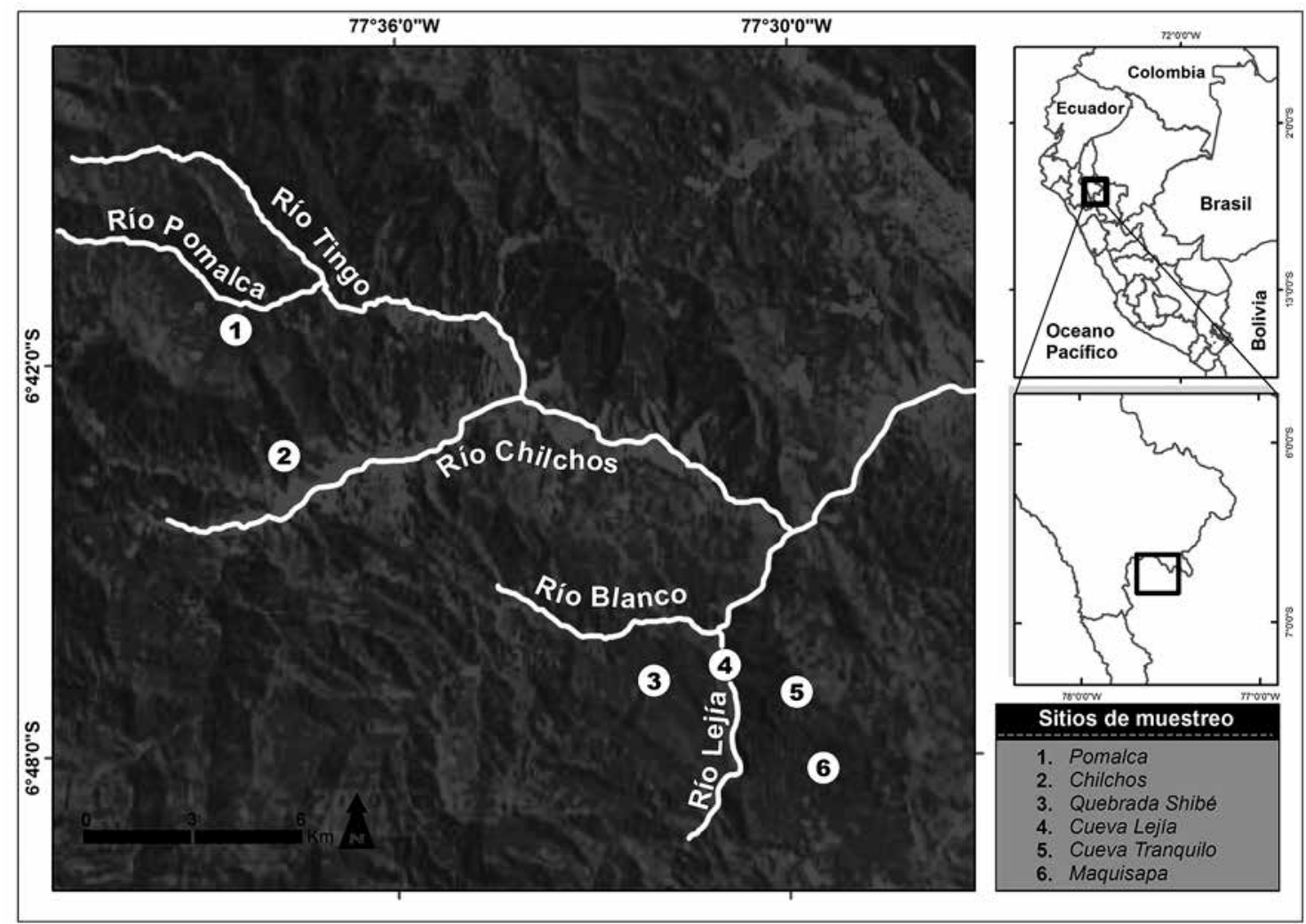

Figura 1. Sitios de muestreo por sectores en bosques de baja y alta perturbación de Los Chilchos. 


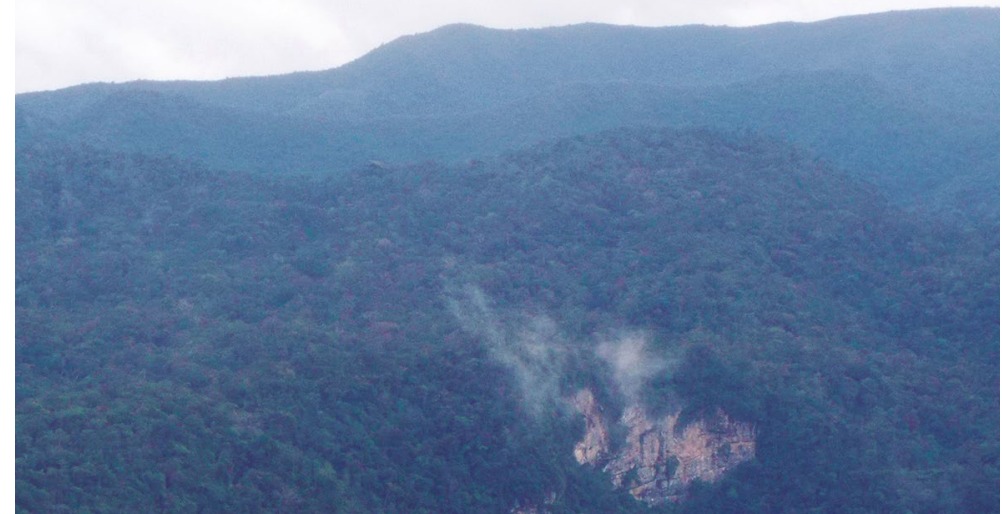

Figura 2. Bosque primario de baja perturbación en el sector de Las Hamacas (al interior del ACP Los Chilchos).

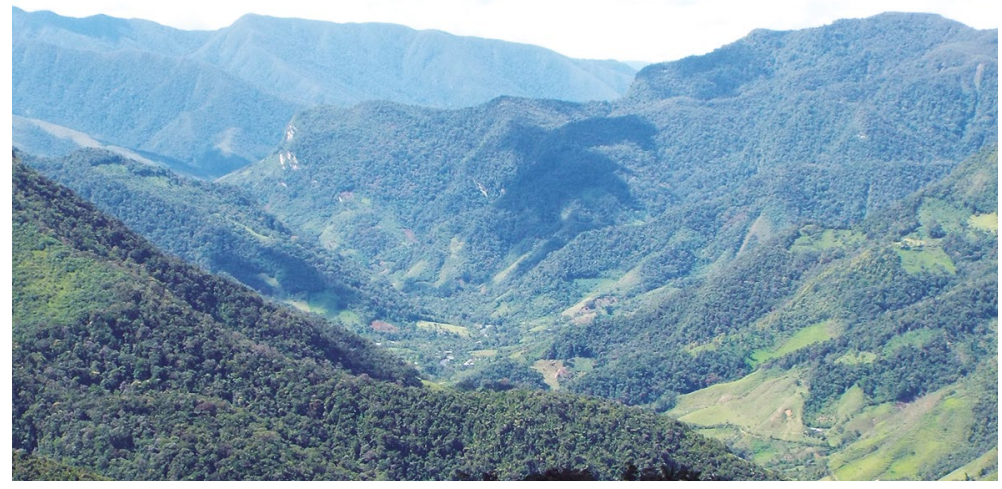

Figura 3. Bosque residual de alta perturbación en el sector de Las Palmas, Los Chilchos.

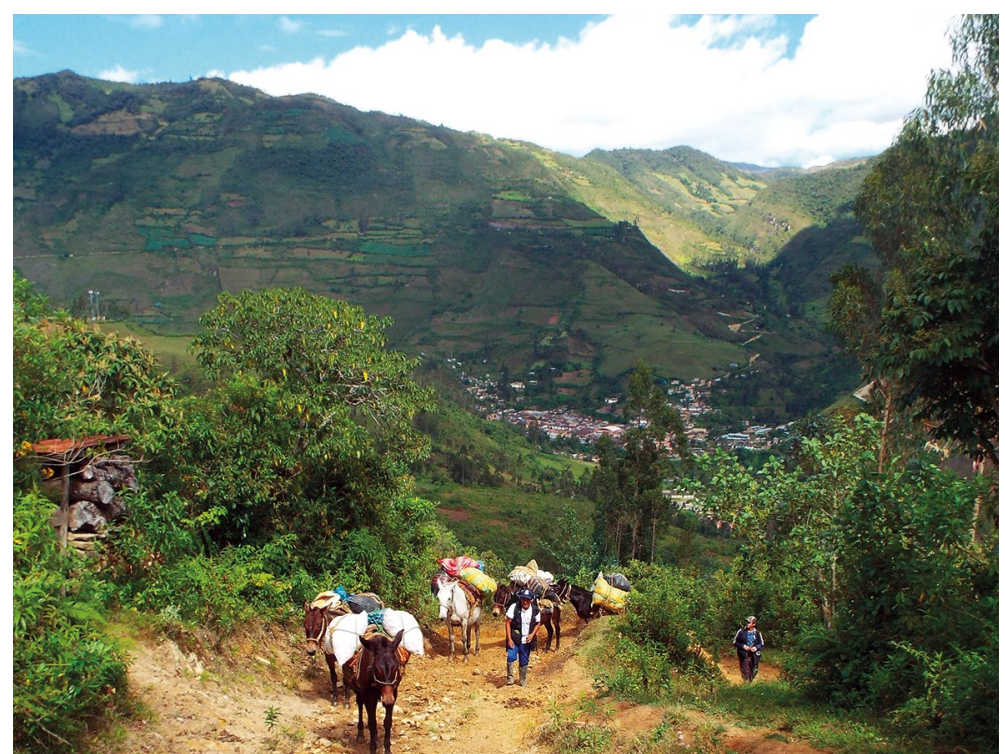

Figura 4. Mulas cargando los materiales de campo y alimentos de Palmira hacia Los Chilchos. 
dedicada a la ganadería y agricultura. En este sector notamos cierta escasez de la fauna mayor, entre ellos los primates.

Es oportuno mencionar que el acceso desde Palmira (anexo del distrito de Leymebamba) hacia el poblado de Los Chilchos, y desde aquí a los sectores y sitios de muestreo es a través de herraduras, por lo que para el traslado de los materiales de campo y alimentos tuvimos que recurrir al uso de acémilas (Fig. 4), único medio de transporte en la mayor parte de los bosques montanos.

\section{Material y métodos}

Censos por transecto lineal.- Para este propósito, en cada sitio de muestreo fueron abiertos cuatro trochas, cuyas longitudes variaron desde 3.0 a $3.5 \mathrm{~km}$. Las trochas tuvieron como punto de inicio nuestro campamento y direccionadas hacia los cuatro puntos cardinales. Para los censos, adicionalmente también hicimos uso de los senderos abiertos por los comuneros para los fines turísticos. Cada transecto fue recorrido de 3 a 4 veces en forma intercalada. Los censos fueron de ida y vuelta desde las 07:00 a 16:00 h (diurno) y desde las 18:30 a 21:00 h (nocturno).

Dos equipos integrados por un investigador y un guía de campo se movilizaron simultáneamente a lo largo de los transectos a una velocidad promedio de $0.7 \mathrm{~km} /$ hora tanto en horario diurno como nocturno. Durante los censos nocturnos no tuvimos mayores contratiempos, por cuanto la fisiografía del terreno facilitó los recorridos, en particular en La Meseta donde el relieve prácticamente es una llanura situada a más de $1500 \mathrm{~m}$ de altitud. Además, la experiencia de los investigadores en censos nocturnos fue muy importante para el registro de datos. Cada vez que un grupo de primates fue avistado se procedió a anotar en la libreta de campo los siguientes datos: hora de contacto, especie, tamaño de grupo (cuando fue posible el conteo completo), distancia perpendicular del primer animal avistado al transecto (medida con una cinta métrica de $30 \mathrm{~m}$ ), altura (en el estrato vertical del bosque), actividad al momento de su detección, presencia de juveniles e infantes, tipo de vegetación circundante y las coordenadas del punto de contacto mediante el uso de un GPS 72H, marca Garmin. A diferencia de las especies de hábito diurno, en el caso de $A$. miconax, la detección de los grupos mayormente fue a través de los ruidos de las ramas ocasionados por los saltos o por la caída de restos de frutos durante la alimentación, así como por la vocalización que emitían al descubrir nuestra presencia, lo que facilitó incluso el conteo de los individuos.

Adicionalmente, se anotaron datos relacionados al nivel de perturbación del bosque y las actividades antrópicas que se desarrollan. En total se han recorrido $402 \mathrm{~km}$ equivalente a 602 horas de censos. De ellos, $282 \mathrm{~km}$ correspondió a los censos diurnos y el resto a los nocturnos (Tabla 2).

Análisis de datos.- Para determinar el tamaño promedio y rango de variación de grupos fue considerado únicamente a los grupos con conteo completo, observados en su mayoría en bosque de baja perturbación, excepto $L$. flavicauda registrada únicamente en bosque de alta perturbación. Para este propósito, cada vez que hubo contacto con primates, procuramos contar a todos los individuos del grupo y cuando fue posible también a los adultos (hembras y machos), juveniles e infantes. El tiempo empleado para el conteo de los individuos dependió del tamaño de grupo y del comportamiento ante nuestra presencia y varió desde 5 hasta más de 15 minutos. Los infantes sin locomoción propia no fueron considerados para determinar el tamaño promedio de grupo.

La densidad poblacional de primates fue estimada únicamente para el bosque de baja perturbación, excepto $A$. miconax que fue la única especie que contó con suficiente registro en bosques de alta perturbación. La densidad fue analizada con el software DISTANCE versión 6.2 (Thomas et al. 2010). La densidad obtenida para todas las especies por medio de este Programa es confiable, no solamente porque el Coeficiente de Variación (CV) resultó menos del $30 \%$, sino porque los datos presentan un ajuste adecuado al modelo establecido ( $p>0.05)$. También se estimó la abundancia relativa, es decir, el número de avistamientos por $10 \mathrm{~km}$ de longitud recorrida tanto en bosques de baja como de alta perturbación. El análisis se hizo mediante una regla de tres simple y está basada sobre el total de longitud recorrida en cada uno de estos bosques. En el caso de $A$. belzebuth, la densidad y abundancia relativa fue estimada en base a 10 grupos de los 17 observados, puesto que asumimos que algunos de ellos fueron registrados hasta en dos oportunidades, al menos así nos indican el tamaño de los grupos observados y las coordenadas de ubicación anotadas al momento de su detección y luego visualizadas en la imagen satelital cuya distancia entre una y otra coordenada en algunos casos no superaban los $300 \mathrm{~m}$.

Tabla 2. Longitud recorrida $(\mathrm{km})$ por sitios de muestreo durante los censos en Los Chilchos.

\begin{tabular}{|c|c|c|c|c|c|}
\hline \multirow[t]{2}{*}{ Sector } & \multirow[t]{2}{*}{ Sitios de muestreo } & \multicolumn{2}{|c|}{ Long. recorrida $(\mathbf{k m})$} & \multicolumn{2}{|c|}{ Esfuerzo (horas censadas) } \\
\hline & & Diurno & Nocturno & Diurno & Nocturno \\
\hline \multirow{2}{*}{ La Meseta } & Cueva Tranquilo & 28 & 11 & 43 & 16 \\
\hline & Maquisapa & 31 & 13 & 40 & 19 \\
\hline \multirow{2}{*}{ Las Hamacas } & Quebrada Shibé & 98 & 45 & 159 & 75 \\
\hline & Cueva Lejia & 12 & 12 & 21 & 17 \\
\hline & Subtotal & 169 & 81 & 263 & 127 \\
\hline \multirow{2}{*}{ Las Palmas } & Pomalca & 68 & 23 & 92 & 33 \\
\hline & Chilchos & 45 & 16 & 68 & 23 \\
\hline \multicolumn{2}{|r|}{ Subtotal } & 113 & 39 & 160 & 56 \\
\hline \multicolumn{2}{|r|}{ Total } & 282 & 120 & 423 & 183 \\
\hline
\end{tabular}




\section{Resultados}

Especies registradas.- En $402 \mathrm{~km}$ de longitud recorrida fueron observados 63 grupos pertenecientes a 5 especies (Tabla 3). De ellos, 50 agrupados en 4 especies se observaron en bosques de baja perturbación y solamente 13 grupos pertenecientes a igual número de especies en bosques de alta perturbación. El mayor registro tanto en bosques de baja como de alta perturbación fue para A. miconax con 31 grupos equivalente al 49\% del total, seguido por $A$. belzebuth (Fig. 5) con 17 grupos registrados, mientras que $L$. flavicauda (Fig. 6) fue la menos observada, con solamente un grupo (Tabla 3).

Como era de esperar, $A$. belzebuth no fue registrado en bosque de alta perturbación, lo que indicaría su alta susceptibilidad a las alteraciones de su hábitat. En el caso de L. flavicauda, la carencia de registros en bosques de baja perturbación indicaría su escasa presencia, quizá por la presencia de $A$. belzebuth, su mayor competidor por el espacio y recursos alimenticios.

Tamaño de grupo.- Grupos más grandes se observaron en Cebus yuracus (tamaño promedio: 17.0 individuos, rango: 12 - 22 individuos), seguido por $A$. belzebuth (tamaño promedio: 14.5 individuos, rango: $6-26$ individuos) (Tabla 4 ). Contrario a los anteriores ocurrió en $A$. seniculus con 4.6 y rango de 3 a 6 individuos y en $A$. miconax, cuyo tamaño promedio de grupo fue 2.6 y rango de 2 a 4 individuos. En L. flavicauda, el grupo observado estuvo compuesto por nueve individuos incluyendo dos infantes sin locomoción propia.

Abundancia relativa y densidad poblacional.- Ambos resultados fueron mayores para las especies que habitan en bosques de bajo nivel de perturbación (Tabla 5). Comparando entre especies, la abundancia relativa fue mayor para $A$. miconax tanto en bosques de baja como de alta perturbación antrópica $(2.59$ y 2.56 grupos $/ 10 \mathrm{~km}$, respectivamente), mientras que la más baja fue para $L$. flavicauda con 0.1 grupos $/ 10 \mathrm{~km}$, seguidos por $A$. seniculus y $C$. yuracus. La densidad más alta fue para $A$. miconax y $A$. belzebuth y la más baja para $A$. seniculus con apenas 3.0 indiv. $/ \mathrm{km}^{2}$ (Tabla 5), resultados que indican su alta representatividad para algunas especies y relativa ausencia para otras, como es el caso de L. flavicauda, especie que no fue registrada durante los censos en bosques de baja perturbación, lo que indicaría su escasa presencia debido a factores diferentes a las condiciones del bosque como se mencionó anteriormente.

\section{Discusión}

Las especies registradas en este estudio coinciden con el reporte de Barrio et al. (2003) para las zonas de la laguna de Los Cóndores y río Chilchos, excepto Ateles chamek. También resulta similar al reportado por Shanee et al. (2013a) para los bosques montanos de la Región Amazonas, pero inferior a los obtenidos por Aquino et al. (2017) para San Martín y en particular para Huánuco, para cuyos bosques se reportan siete especies. Con referencia a $A$. chamek, citado por Barrio et al. (2003) para Los Chilchos, exploraciones recientes indicarían la ausencia de esta especie en esta parte de la Amazonía peruana. $\mathrm{Al}$ respecto, J. Dignum (citado por Shanee et al. 2013a) sostiene que se debe a una identificación errónea basado en el pelaje más oscuro de algunos grupos de $A$. belzebuth que son confundidos con $A$. chamek, opinión que es compartida por Aquino et al (2018), quienes mencionan que se trata de un error considerar la presencia de esta especie en bosques montanos al oeste del río Huallaga, por cuanto mucha gente conocen como "maquizaoa

Tabla 3. Proporción (\%) y número $(\mathrm{N})$ de grupos de primates observados por nivel de perturbación en Los Chilchos.

\begin{tabular}{|c|c|c|c|c|c|c|}
\hline \multirow{3}{*}{ Especies } & \multicolumn{4}{|c|}{ Nivel de perturbación } & \multicolumn{2}{|c|}{ Total } \\
\hline & \multicolumn{2}{|c|}{ Bajo } & \multicolumn{2}{|c|}{ Alto } & \multirow[b]{2}{*}{$\mathrm{N}$} & \multirow[b]{2}{*}{$\%$} \\
\hline & $\mathrm{N}$ & $\%$ & $\mathrm{~N}$ & $\%$ & & \\
\hline Ateles belzebuth & 17 & 34 & & & 17 & 27 \\
\hline Lagothrix flavicauda & & & 1 & 8 & 1 & 2 \\
\hline Alouatta seniculus & 6 & 12 & 1 & 8 & 7 & 11 \\
\hline Cebus yuracus & 6 & 12 & 1 & 8 & 7 & 11 \\
\hline Aotus miconax & 21 & 42 & 10 & 76 & 31 & 49 \\
\hline Total & 50 & 100 & 13 & 100 & 63 & 100 \\
\hline
\end{tabular}

Tabla 4. Tamaño y rango de variación de grupos en primates observados en Los Chilchos.

\begin{tabular}{|c|c|c|c|c|c|}
\hline \multirow{2}{*}{ Especies } & \multicolumn{2}{|c|}{ Tamaño de grupo } & \multicolumn{2}{|c|}{ Número de grupos } & \multirow{2}{*}{ Rango en otras áreas } \\
\hline & Promedio \pm DS & Rango & Total & Con conteo completo & \\
\hline Ateles belzebuth & $14.5 \pm 7.2$ & $6-26$ & 17 & 6 & $6-18^{a}$ \\
\hline Lagothrix flavicauda & & 9 & 1 & 1 & $3-19^{b}$ \\
\hline Alouatta seniculus & $4.6 \pm 1.1$ & $3-6$ & 7 & 5 & $3-9^{c}$ \\
\hline Cebus yuracus & $17 \pm 5$ & $12-22$ & 7 & 2 & $9-13^{d}$ \\
\hline Aotus miconax & $2.6 \pm 0.7$ & $2-4$ & 31 & 12 & $2-5^{\mathrm{e}}$ \\
\hline
\end{tabular}

${ }^{a}$ Northeast Peruvian Amazon (Aquino et al.2016b), ${ }^{\mathrm{b}}$ La Esperanza (Shanee \& Shanee 2011a), ${ }^{\mathrm{CH}}$ uánuco (Aquino et al. 2015), ${ }^{\mathrm{d}} \mathrm{Huánuco-San} \mathrm{Martín} \mathrm{(Aquino} \mathrm{et} \mathrm{al.}$ 2017).

eAbra Patricia-Alto Nieva (Cornejo et al. 2008). 


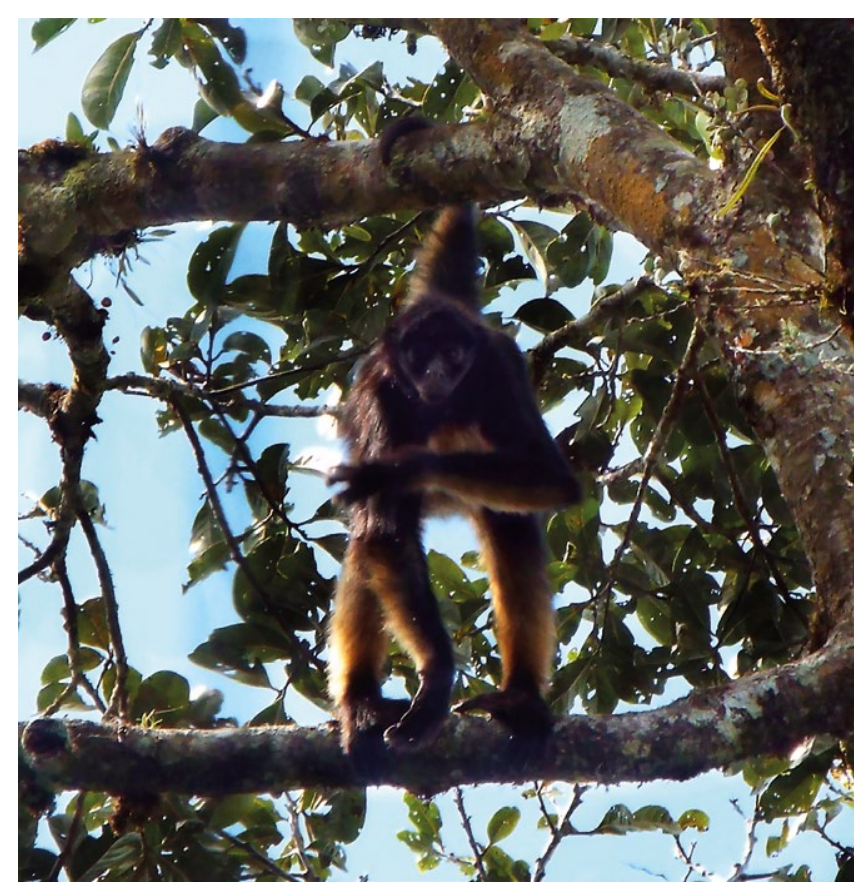

Figura 5. Ejemplar adulto de $A$. belzebuth observado en La Meseta (fuera del ACP Los Chilchos).

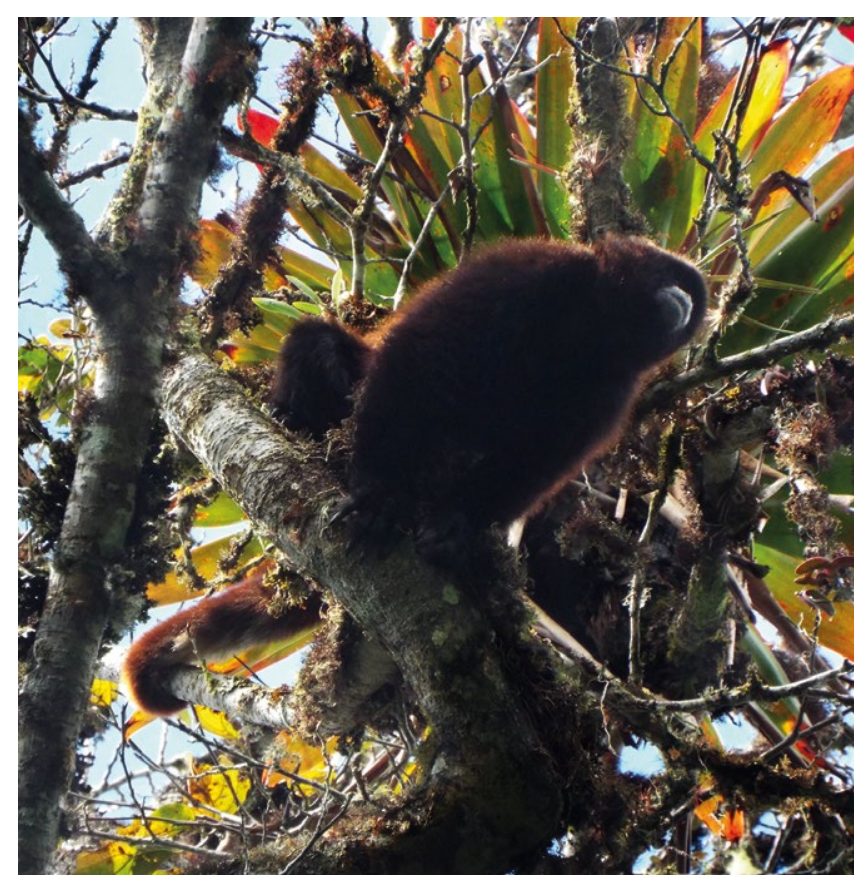

Figura 6. Ejemplar adulto de Lagothrix flavicauda observado en Pomalca, Los Chilchos.

Tabla 5. Abundancia relativa y densidad poblacional de primates en bosques de bajo y alto nivel de perturbación en Los Chilchos.

\begin{tabular}{|c|c|c|c|}
\hline \multirow{2}{*}{ Especies } & \multicolumn{2}{|c|}{ Nivel de perturbación } & \multirow{2}{*}{ Otras áreas } \\
\hline & Bajo & Alto & \\
\hline \multicolumn{4}{|l|}{ Ateles belzebuth } \\
\hline \# de encuentros/10 km de transecto recorrido & 0.6 & & $0.3-0.7^{\mathrm{a}}$ \\
\hline Individuos $/ \mathrm{km}^{2}$ & 13.0 & & $3.6-23^{\mathrm{a}}$ \\
\hline$\% \mathrm{CV}$ & 29.16 & & \\
\hline Chi cuadrado & 0.24 & & \\
\hline \multicolumn{4}{|l|}{ Lagothrix flavicauda } \\
\hline \# de encuentros $/ 10 \mathrm{~km}$ de transecto recorrido & & 0.1 & $0.5^{\mathrm{b}}$ \\
\hline Individuos $/ \mathrm{km}^{2}$ & & & $8.27-9.26^{c}$ \\
\hline \multicolumn{4}{|l|}{ Alouatta seniculus } \\
\hline \# de encuentros/10 km de transecto recorrido & 0.36 & 0.1 & $0.24^{\mathrm{b}}$ \\
\hline Indiv. $/ \mathrm{km}^{2}$ & 3.0 & & $0.5^{\mathrm{b}}$ \\
\hline$\% \mathrm{CV}$ & 13.4 & & \\
\hline Chi cuadrado & 0.25 & & \\
\hline \multicolumn{4}{|l|}{ Cebus yuracus } \\
\hline \# de encuentros/10 km de transecto recorrido & 0.36 & 0.1 & $0.1^{\mathrm{b}}$ \\
\hline Individuos $/ \mathrm{km}^{2}$ & 9.73 & & $4.5^{\mathrm{b}}$ \\
\hline$\% \mathrm{CV}$ & 8.22 & & \\
\hline Chi cuadrado & 0.41 & & \\
\hline \multicolumn{4}{|l|}{ Aotus miconax } \\
\hline \# de encuentros/10 km de transecto recorrido & 2.59 & 2.56 & n.d \\
\hline Individuos $/ \mathrm{km}^{2}$ & 26.23 & 24.5 & n.d. \\
\hline$\% \mathrm{CV}$ & 16.66 & 9.9 & \\
\hline Chi cuadrado & 0.46 & 1.0 & \\
\hline
\end{tabular}

Fuente: aAquino et al. (2016b), bAquino et al.(2015), 'Shanee \& Shanee (2011a). n.d.: No disponible. 
negra" a los ejemplares de $A$. belzebuth que tiene el pelaje del dorso, incluyendo la cabeza de color negro intenso y el lado ventral, en particular el pecho de color negruzco.

De las especies hasta ahora registradas para los bosques montanos, Sapajus macrocephalus fue la única que no se observó en ninguno de los sitios muestreados. Al respecto, Mittermeier et al. (1975) y Shanee et al. (2013a) tampoco lograron registrar a este primate en lugares explorados correspondiente a la Región Amazonas, por lo que estos bosques no formarían parte de su área de distribución geográfica, excepto el norte, donde Butchart et al. (1995), habría observado ocho grupos durante sus exploraciones en la Cordillera Colán, no obstante, sería de vital importancia confirmar la existencia de este primate en esta parte de la Región Amazonas.

Lagothrix flavicauda no fue registrada en Las Hamacas y La Meseta, contrastando así con Barrio et al. (2003), quienes aseguran haber observado un grupo en un acantilado frente al Río Blanco, cerca de Las Hamacas, lo que indicaría que en estos sectores habría una pequeña población, quizá similar a Las Palmas, donde se observó un grupo de 9 individuos, lo cual no quiere decir que no existan otros grupos. En efecto, uno de los autores (J. Dignum) observó en el 2009 un grupo de aproximadamente 15 individuos, quien también tuvo encuentros con individuos solitarios. El grupo observado en este estudio estuvo integrado por 1 macho adulto (que se caracterizó por presentar un mechón de pelos de color amarillo cremoso entre las piernas), 3 hembras adultas, 3 juveniles y 2 infantes sin locomoción propia (cargados por sus respectivas progenitoras). En el caso de los juveniles, no fue posible identificar el sexo debido a su tamaño relativamente pequeño y porque al momento del contacto, hubo neblina y garúas que dificultaron una mejor visualización. La probable escasa presencia de este primate en Las Hamacas y La Meseta podría estar relacionado con la mayor abundancia de $A$. belzebuth, potencial competidor por el hábitat y recursos alimenticios. Por otro lado, la ausencia de registros de A. belzebuth en Las Palmas es justificable, por cuanto son los más susceptibles a la caza y alteración de su hábitat (Aquino et al. 2016a), coincidiendo así con Roosmalen \& Klein (1988), quienes sostienen que los Ateles en general han demostrado su poca o nula adaptabilidad a la perturbación humana.

El rango y tamaño promedio de los grupos de A. belzebuth observados entre las Hamacas y La Meseta fueron más grandes a los reportados para el río Pucacuro (Aquino et al. 2000) y río Samiria (Aquino \& Bodmer 2006), ambos en la Amazonía baja y sometidos a una alta presión de caza. También superaron en tamaño a los obtenidos para el Curaray-Nashiño y CurarayArabela, considerados como de baja perturbación antrópica (Aquino et al., 2013) y bosques nororientales de la Amazonía peruana con moderada a baja perturbación (Aquino et al. 2016b).

Con respecto a L. flavicauda, observado en bosques de alta perturbación antrópica, no permite realizar mayores comparaciones; no obstante, podemos sostener que resultó menor a los reportados para el Río Abiseo (Leo Luna 1982), Bosque de Protección Alto Mayo (DeLuycker 2007), La Esperanza (Shanee \& Shanee 2011a), Chontayacu-Miraflores (Aquino et al. 2015) y Huánuco (Aquino et al., 2016a), lugares donde los grupos variaron desde un mínimo de 6 hasta más de 23 individuos. Sin embargo, fue ligeramente mayor al proporcionado por Cornejo (2007) para el Área de Conservación Privada Abra Patricia-Alto
Nieva, donde el tamaño promedio de grupo fue estimado en 5.53 y rango de 2 a 7 individuos.

El tamaño promedio de grupo de $A$. seniculus, estimado en este estudio resulta similar a los registrados para San Martín y Huánuco (Aquino et al. 2017), donde los grupos variaron de 2 a 5 , con un tamańo promedio de 4 y 3 individuos, respectivamente. Sin embargo, fue menor al reportado para los bosques sub-andinos de Colombia (Morales-Jiménez 2002), cuyos grupos fluctuaron de 2 a 9 y un promedio de 6 individuos También resulta menor al de Miraflores (Aquino et al. 2015), donde se observaron grupos mayores a nueve individuos. Con respecto a C. yuracus, el rango y tamańo promedio del grupo estimado para el bosque de baja perturbación fue mayor que los reportados para San Martín, pero similar a Huánuco (Aquino et al. 2017), donde los grupos en algunos casos superaron los 15 individuos.

Finalmente, el rango del tamaño de los grupos de $A$. miconax observados en Los Chilchos coincide con los reportes de Butchart et al. (1995) para la Cordillera Colán y de Cornejo et al. (2008) para Abra Patricia y Huiquilla, quienes observaron grupos entre 2 a 3 y 2 a 4 individuos, respectivamente. Sin embargo, fue menor a los proporcionados por Shanee \& Shanee (2011b) para La Esperanza, cuyo rango fue de 2 a 6 individuos, pero resulta cercano al obtenido por Hirche et al. (2017) para Aotus cf. murinus en bosque subandino de Colombia, cuyo tamaño promedio fue estimado en 2.2 y rango de 1 a 5 individuos.

La abundancia relativa y densidad poblacional de $A$. belzebuth estimada en este estudio fue ligeramente menor a los obtenidos por Aquino et al. $(2013,2016 \mathrm{~b})$ para los bosques del noreste del Perú con baja y moderada perturbación humana, pero fue más alto al reportado para el Río Pucacuro (Aquino et al. 2000) y Río Samiria (Aquino \& Bodmer 2006), ambas sometidas a una alta presión de caza. Por otro lado, la abundancia relativa estimada para L. flavicauda resultó mucho menor al reportado por Aquino et al. (2015) para Yanajanca-Cocalito que fue de 0.5 grupos $/ 10$ $\mathrm{km}$ y bosques montanos de Huánuco (1.8 indiv. $/ 10 \mathrm{~km}$ ).

Con respecto a $A$. seniculus, la abundancia relativa estimada para el bosque de baja perturbación en Chilchos superó ligeramente al obtenido para San Martín y Huánuco (Aquino et al. 2017), pero la densidad fue menor al de Huánuco que fue estimada en 5 indiv. $/ \mathrm{km}^{2}$ (Aquino et al. 2015). En el caso de C. $y u$ racus, tanto la abundancia relativa como la densidad poblacional estimadas para el bosque de baja perturbación de Los Chilchos, fueron más altas que las reportadas para Chontayacu-Miraflores (Aquino et al. 2015) y bosques montanos de San Martín y Huánuco (Aquino et al. 2017), lugares donde se encuentran bosques residuales esencialmente primario, pero la presión de caza al parecer es muy alta para el control de poblaciones.

En lo que concierne a $A$. miconax, estamos en condiciones de asegurar que no existen antecedentes sobre abundancia relativa y densidad poblacional, por lo que no es posible realizar comparaciones, salvo con otras especies con hábitats similares, como es el caso de Aotus cf. lemurinus que habita en bosques subandinos de Colombia, para cuya especie Hirche et al. (2017) usando la misma metodología estimaron en 113 indiv. $/ \mathrm{km}^{2}$, diferencia abismal si comparamos con nuestros resultados. De acuerdo con los autores, esa alta densidad podría estar relacionada con la reducción de los competidores debido a la extinción local de otros primates u otras especies que son menos tolerantes a la 
reducción y fragmentación del bosque y a las amenazas sinérgicas como la caza. No obstante, resultaron muy cercanos a la estimada por Aquino y Encarnación (1988) para A. nancymaae en bosques no inundables $\left(24.2\right.$ indiv. $\left./ \mathrm{km}^{2}\right)$, pero mayor al de A. vociferans en bosques similares $\left(7.9\right.$ indiv. $\left./ \mathrm{km}^{2}\right)$.

\section{Agradecimientos}

Agradecemos a Primate Action Fund/ Conservation International, Primate Conservation Inc. y Apenheul Primate Conservation Trust (APCT), por los fondos proporcionados, que permitió llevar a cabo la primera evaluación de primates en Los Chilchos, Región Amazonas. Nuestro agradecimiento a los guías de campo, en particular a Domingo Huamán por su constante participación durante la instalación de los campamentos, apertura de trochas y censos en los sitios de muestreo y a los hermanos Joaquín y Ernesto Briones por su experiencia y vasto conocimiento del área de estudio. Finalmente, hacemos extensivo nuestra gratitud a la ONG Ucumari, responsable de la organización y coordinación para el inventario en Los Chilchos y a la Municipalidad Distrital de Leymebamba, por el permiso otorgado para el inventario en Los Chilchos.

\section{Literatura citada}

Aquino R. \& F. Encarnación. 1988. Population densities and geographic distribution of night monkeys (Aotus nancymae and Aotus vociferans) (Cebidae: Primates) in northeastern Peru. American Journal of Primatology 14 (4): 375 - 381. https:// doi.org/10.1002/ajp.1350140407

Aquino R. \& F. Encarnacion. 1994. Primates of Peru/ Los Primates del Perú. Primate Report 40: 1-130.

Aquino R., R. E. Bodmer \& E. Pezo. 2000. Evaluación de primates en la Cuenca del río Pucacuro. En: J. Espinoza, F. San Martín y E. Montoya, eds. La Primatología en el Perú Vol.2. Master Graf. Editores, Lima. Pp. 92-100.

Aquino R. \& R. E. Bodmer. 2006. Distribución y abundancia de Ateles belzebuth E. Geoffroy y Ateles chamek Humboldt (Cebidae: Primates) en la Reserva Nacional Pacaya Samiria, Perú. Revista Peruana de Biología 13: 103-106. DOI: http:// dx.doi.org/10.15381/rpb.v13i1.1769.

Aquino R., F. Cornejo, E. Pezo \& E. Heymann. 2013. Distribution and abundance of white-fronted spider monkeys, Ateles belzebuth (Atelidae) and threats to their survival in Peruvian Amazonia. Folia Primatologica 84(1): 1-10. http://dx.doi. org/10.1159/000345549.

Aquino R., R. Zárate, L. López, et al. 2015. Current status and threats to Lagothrix flavicauda and other primates in montane forest of the Región Huánuco. Primate Conservation 29: 1-11. http://dx.doi.org/10.1896/052.029.0111

Aquino R., G. García \& E. Charpentier. 2016a. Distribution and current status of the peruvian yellow-tailed woolly monkey (Lagothrix flavicauda) in montane forests of the Region Huánuco. Primate Conservation 30: 31-37.

Aquino R., L. López, G. García \& E. Charpentier. 2016b. Conservation status and threats to atelids in the northeastern Peruvian Amazon. Primate Conservation 30: 21-29.

Aquino R., G. García, E. Charpentier \& L. López. 2017. Estado de conservación de Lagothrix flavicauda y otros primates en bosques montanos de San Martín y Huánuco, Perú. Revista Peruana de Biología 24: 25-34. http://dx.doi.org/10.15381/ rpb.v24i1.13101.

Aquino R., L. López, H. Gálvez \& S. Díaz. 2018. Current status of Ateles belzebuth in montane forests of the Peruvian Amazon. Primate Conservation 32:1-11. http://www.primate-sg.org/ storage/pdf/PC32_Aquino_et_al_A_belzebuth_Peru.pdf

Barrio J., C. Mendoza \& P. Venegas. 2003. Inventario biológico preliminar de la Cordillera Nororiental, Zonas de laguna de Los Cóndores y Río Chilchos. Reporte final de la evaluación de fauna silvestre ejecutada en noviembre-diciembre 2003. ONG UCUMARI, Fundación Holandesa Stichting DNP. $30 \mathrm{pp}$.
Butchart S. H. M., R. Barnes, C. W. N. Davies, et al. 1995. Observations of two threatened primates in the Peruvian Andes. Primate Conservation 16: 15-19.

Cornejo F. M. 2007. Estado de conservación de Oreonax flavicauda "Mono Choro Cola Amarilla" en el Área de Conservación Privada Abra Patricia-Alto Nieva. Tesis Título Profesional de Bióloga, Facultad de Ciencias Biológicas, Universidad Nacional Mayor de San Marcos, Lima. 44pp.

Cornejo F. M., R. Aquino \& C. Jiménez. 2008. Notes on the natural history, distribution and conservation status of the Andean night monkey, Aotus miconax Thomas, 1927. Primate Conservation 23: 1-4. https://doi.org/10.1896/052.023.0101

Defler T. R. 2003. Primates de Colombia. Conservation International Colombia. 543 pp.

Defler T. R. 2010. Historia natural de los primates colombianos. 2nd edn. Universidad Nacional de Colombia, Bogotá, D.C. $612 \mathrm{pp}$.

DeLuycker A. M. 2007. Notes on the yellow-tailed woolly monkey (Oreonax flavicauda) and its status in the Protected Forest of Alto Mayo, Northern Peru. Primate Conservation 22: 41-47. http://dx.doi.org/10.1896/052.022.0102

Hirche A., A. Jimenez, N. Roncancio-Duque \& H. Ansorge. 2017. Population density of Aotus cf. lemurinus (Primates: Aotidae) in a Subandean forest patch on the eastern slopes of the western Andes, Region of Dapa, Yumbo, Valle del Cauca, Colombia. Primate Conservation 31: 1-7.

IUCN (International Union for Conservation of Nature).2017. IUCN Red List of Threatened Species. Version 2017-3. Website:www.iucnredlist.org>. Accessed: 13 Marzo 2018.

Leo Luna M. 1980. First field study of the yellow-tailed woolly monkey. Oryx 15: $386-389$.

Leo Luna M. 1982. Estudio Preliminar sobre la Biología y Ecología del Mono Choro de Cola Amarilla Lagothrix flavicauda (Humboldt 1812). Tesis Bióloga, Universidad Nacional Agraria La Molina, Lima, Perú.

Leo Luna M. 1984. The effect of hunting, selective logging, and clearcutting on the conservation of the Yellow-tailed Woolly Monkey (Lagothrix flavicauda). Tesis Magister, University of Florida, Gainesville, FL.

Leo Luna M. 1987. Primate conservation in Peru: a case study of the Yellow-tailed Woolly Monkey. Primate Conservation 8: 122-123.

Mittermeier R. A., H. de Macedo-Ruíz \& B. A. Luscombe. 1975. A woolly monkey rediscovered in Peru. Oryx 13: 41- 46.

Morales-Jiménez A. 2002. Densidad de los monos aulladores (Alouatta seniculus) en un bosque subandino, Risaralda, Colombia. Neotropical Primates 10: 141-144.

Roosmalen M.G.M, \& L.L. Klein. 1998. The Spider Monkeys, genus Ateles. En R.A. Mittermeier, A.B. Rylands, A.F. CoimbraFilho \& G.A.B. de Fonseca, eds. Ecology behavior of Neotropical Primates. World Wildlife Found, Washington, DC. Pp. 455-537.

Shanee S., N. Shanee \& A. M. Maldonado. 2007a. Conservation assessment and planning for the yellow tailed woolly monkey (Oreonax flavicauda) in Peru. Wildlife Biology in Practice 3: 73-82.

Shanee N., S. Shanee \& A. M. Maldonado. 2007b. Interspecific association between Oreonax and Ateles, Amazonas, Peru. Neotropical Primates 14: 34-35. https://doi. org/10.1896/044.014.0114

Shanee S., N. Shanee \& A. M. Maldonado. 2008. Distribution and conservation status of the Yellow-tailed Woolly Monkey (Oreonax flavicauda, Humboldt 1812) in Amazonas and San Martín, Peru. Neotropical Primates 14: 115-119.

Shanee S. 2011. Distribution survey and threat assessment of the yellow-tailed woolly monkey (Oreonax flavicauda; Humboldt 1812), Northeastern Peru. International Journal of Primatology 32:691-707. 691. http://dx.doi.org/10.1007/ s10764-011-9495-x

Shanee S. \& N. Shanee. 2011 a. Population density estimates of the Critically Endangered yellow-tailed woolly monkeys (Oreonax flavicauda) at La Esperanza, northeastern Peru. International Journal of Primatology 32: 878-888. http:// dx.doi.org/10.1007/s10764-011-9507-x 
Shanee S. \& N. Shanee. 2011b. Observations of terrestrial behavior in the Peruvian night monkey (Aotus miconax) in an anthropogenic landscape, La Esperanza, Peru. Neotropical Primates 18(2): 55-58. https://doi.org/10.1896/044.018.0205

Shanee N. 2012. The dynamics of threats and conservation efforts for the tropical Andes hotspot in Amazonas and San Martin, Peru. PhD Thesis, DICE, Kent University, vii+426pp.

Shanee S., N. Shanee \& N. Allgas-Marchena. 2013a. Primate surveys in the Marañón-Huallaga landscape, Northern Peru with notes on conservation. Primate Conservation 27: 3-11. http://dx.doi.org/10.1896/052.027.0114.

Shanee S., N. Allgas \& N. Shanee. 2013b. Preliminary observations on the behavior and ecology of the Peruvian night monkey (Aotus miconax: Primates) in a remnant cloud forest patch, north eastern Peru. Tropical Conservation Science 6(1): 138-148. https://doi.org/10.1177/194008291300600104.
Shanee S. 2014. Ranging behavior, daily path lengths, diet and habitat use of yellow-tailed woolly monkeys (Lagothrix flavicauda) at La Esperanza, Peru. En: T. R. Defler, P. R. Stevenson, eds. The Woolly Monkey, Developments in Primatology: Progress and Prospects. Springer New York. Pp. 167-185. DOI 10.1007/978-1-4939-0697-0_10.

Shanee S., N. Allgas, N. Shanee \& N. Campbell. 2015. Distribution, ecological niche modelling and conservation assessment of the Peruvian Night Monkey (Mammalia: Primates: Aotidae: Aotus miconax Thomas, 1927) in northeastern Peru, with notes on the distributions of Aotus spp. Journal of Threatened Taxa 7(3): 6947-6964. http://dx.doi.org/10.11609/ JoTT.o4184.6947-64.

Thomas L., S.T. Buckland, E.A. Rexstad, J.L. Laake, et al. 2010. Distance software: design and analysis of distance sampling surveys for estimating population size. Journal of Applied Ecology 47: 5-14. https://doi.org/10.1111/j.13652664.2009.01737. 